Revista Eletrônica em Gestão, Educação e Tecnologia Ambiental Santa

Maria, v. 20, n. 1, jan.-abr. 2016, p. 32-39

Revista do Centro de Ciências Naturais e Exatas - UFSM

ISSN : 22361170

\title{
Modelagem matemática em análises de riscos geomorfológicos: um estudo de caso da Rocinha/RJ - Brasil
}

\author{
Mathematical modeling of geomorphological risk analysis: a case study of Rocinha/RJ - Brazil \\ Maurício Kunz ${ }^{1}$, Bruno Macali da Luz ${ }^{1}$, Alcindo Neckel², Luciano Faustinoni ${ }^{3}$ \\ ${ }^{1}$ Acadêmico da Escola de Arquitetura e Urbanismo, Faculdade Meridional - IMED, Passo Fundo, RS, Brasil \\ ${ }^{2}$ Doutor em Geografia, Escola de Arquitetura e Urbanismo, Faculdade Meridional - IMED, Passo Fundo, RS, Brasil \\ ${ }^{3}$ Mestre em Matemática, Escola de Engenharia Civil, Faculdade Meridional - IMED, Passo Fundo, RS, Brasil
}

\begin{abstract}
Resumo
Cada vez mais, as interferências da ocupação humana no ambiente estão ligadas às ocorrências de desastres naturais em áreas de risco. A inexistência de planejamento urbano, a ocupação humana em áreas irregulares e impróprias para fins habitacionais, a remoção da vegetação e a falta de controle por parte do poder público são aspectos da base desse problema que afeta a população brasileira especialmente em períodos com elevados níveis pluviométricos. Ao remover a vegetação dessas áreas, o solo torna-se mais suscetível à infiltração de água, que desestabiliza a parte mais superficial do solo, resultando em solapamentos. Esta pesquisa tem como objetivo demonstrar que, através do uso de geotecnologias, é possível identificar áreas de risco do Bairro da Rocinha, na cidade do Rio de Janeiro (RJ-Brasil), sugerindo possíveis prevençães para futuros desastres. Com isso, permite-se a execução de medidas preventivas. As ferramentas de geoprocessamento, a coleta e a análise de informações geográficas facilitam o processo de tomada de decisões a partir das informações sobre as áreas analisadas, o que pode servir como base para vários estudos de investigação.
\end{abstract}

Palavras-chave: Vulnerabilidade. Risco. Geomorfologia. Solapamento.

\begin{abstract}
Increasingly interference of human occupation in the environment are connected with the occurrences of natural disasters in hazardous areas. The lack of urban planning, along with human occupation in improper uneven areas for residential purposes, the removal of vegetation and the lack of government control are aspects that are part of the root of this problem affecting the population especially in periods that elevate the rainfall levels. By removing vegetation such areas, the soil becomes more susceptible to water ingress which destabilizes the most part of the soil surface, resulting in undermining. This research has the general objective to demonstrate that, through the use of geo possible to identify risk areas of Rocinha (RJ-Brazil), suggesting possible preventions for future disaster. Thus, to allow the execution of preventive measures. The geoprocessing tools, the collection and analysis of geographic information facilitate the process of decision-making from the information on the areas analyzed, which can serve as a basis for various research studies.
\end{abstract}

Keywords: Vulnerability. Risk. Geomorphologic. Undermining. 


\section{Introdução}

Os solapamentos de encostas, segundo Andrade (2002), Viana, Aquino, Munoz (2009) e Robaina, Pedrosa, Bazzan e Reckziegel (2011), têm aumentado consideravelmente nas últimas décadas, deixando a população exposta aos riscos naturais no ambiente. Isso se dá principalmente em razão do aumento de domicílios em áreas periféricas.

A Prefeitura da Cidade do Rio de Janeiro (2012), em Ata Pública do Conselho Estratégico de Informações da Cidade, decidiu, em 12 de setembro de 2012, não utilizar o termo favela para classificar áreas periféricas habitadas de forma ilegal. Assim, passou a denomina-as de aglomerados subnormais. Nesse sentido, o Instituto Brasileiro de Geografia e Estatística (IBGE, 2010, p. 1; IBGE, 2015), conceitua aglomerado subnormal como o "conjunto constituído por no mínimo 51 unidades habitacionais (barracos, casas etc.), ocupando - ou tendo ocupado - até período recente, terreno de propriedade alheia (pública ou particular)". Essa forma de ocupação pode ser caracterizada como sendo feita, "em geral, de forma desordenada e densa; e carente, em sua maioria, de serviços públicos e essenciais".

As habitações construídas nessas áreas encontram-se geralmente em condição de risco, expondo os moradores. Para Robaina, Pedrosa, Bazzan e Reckziegel (2011, p. 16), os riscos causados pelos "movimentos de massa em encostas são um dos problemas urbanos que mais têm aumentado nos últimos anos, sobretudo em períodos chuvosos". Esses deslizamentos são agravados em função da construção de habitações em encostas acentuadas, em que a estrutura do solo se fragiliza em períodos de elevados índices pluviométricos, aumentando sua carga, o que faz o solo se deslocar sobre a superfície rochosa (solapamento).

A importância de entender o risco geomorfológico que os habitantes da Rocinha estão sujeitos, direciona ao objetivo geral dessa pesquisa, que busca caracterizar e avaliar as áreas de risco de solapamentos de encostas deste aglomerado subnormal da Rocinha, no Rio de Janeiro, a partir do levantamento planialtimétrico, obtido de dados georreferenciados e tratados com o software Surfer 12 e com a aplicabilidade de modelos matemáticos de evolução do terreno. Esse estudo se justifica a partir do pressuposto de que as pessoas, ao morarem em áreas de risco, ficam vulneráveis aos deslizamentos e colocam-se à mercê do acaso. Nem mesmo a experiência adquirida com os deslizamentos anteriores as livram da exposição e das tragédias que um novo desastre pode provocar.

Para Marandola JR., Marques, Paula e Cassaneli (2013, p. 51), a solução para isso poderia ser "a elaboração de planos e gastos, quanto às intervenções para 'regularização' das áreas vulneráveis, normalmente pautados em medidas como impermeabilização do solo", seguido da construção de muros de espera, integrados com o dimensionamento das águas pluviométricas através de calhas. Nesse sentido, deve ser levada em conta fatores como "conformação da encosta, retaludamento, demolição e reconstrução parcial da instalação e, em última instância, remoção da moradia", conferindo "ações setoriais, incorporando todo o contexto dinâmico no qual a vida dessas pessoas está inserida".

Entretanto, para propor soluções para a mitigação dos riscos naturais em relação às habitações de encostas, também devem ser estudadas as características físicas do local. Carmo, Souto, Duarte, Mesquita (2015, p. 5928) sugerem a aplicabilidade das "técnicas de Geoprocessamento" em relação ao diagnóstico da área, já que elas "têm favorecido e facilitado o estudo da suscetibilidade a riscos geológicos, podendo definir assim uma melhor gestão territorial, ou seja, auxiliando o processo de tomada de decisão a respeito da ocupação de espaços e seus usos".

$\mathrm{O}$ entendimento acerca dos riscos geomorfológicos a que os habitantes deste aglomerado subnormal da Rocinha (RJ) estão expostos foi concentrado com o auxílio da ferramenta de geoprocessamento no software Surfer 12. Com isso, gerou-se um modelo digital de elevação do terreno, 
modelo pelo qual se podem averiguar as características físicas (geomorfologia, declividade) do terreno. Além desse modelo, foram buscadas informações referentes ao tipo de solo predominante.

Assim, analisando os dados planialtimétricos e o solo da Rocinha, pretende-se identificar e sugerir soluções para essa área ocupada que apresenta risco de deslizamentos. Intende-se tornar possível determinar as áreas com maior prioridade de remoção e reassentamento dos moradores.

\section{Procedimentos metodológicos}

O aglomerado subnormal da Rocinha localiza-se no Estado do Rio de Janeiro (RJ-Brasil) (Figura 1), segundo dados do Instituto Brasileiro de Geografia e Estatística (IBGE), possui 23.352 domicílios ocupados, com uma população de 73.410 habitantes, conforme o Censo Demográfico (2010). De acordo com esses dados quantitativos, sobre o total populacional residente na Rocinha estima-se que, com as recusas de moradores que não quiseram responder ao censo e as residências com moradores ausentes, pode-se chegar à estimativa de 98.319 habitantes vivendo em condições de riscos naturais e condições precárias (IBGE, 2015).

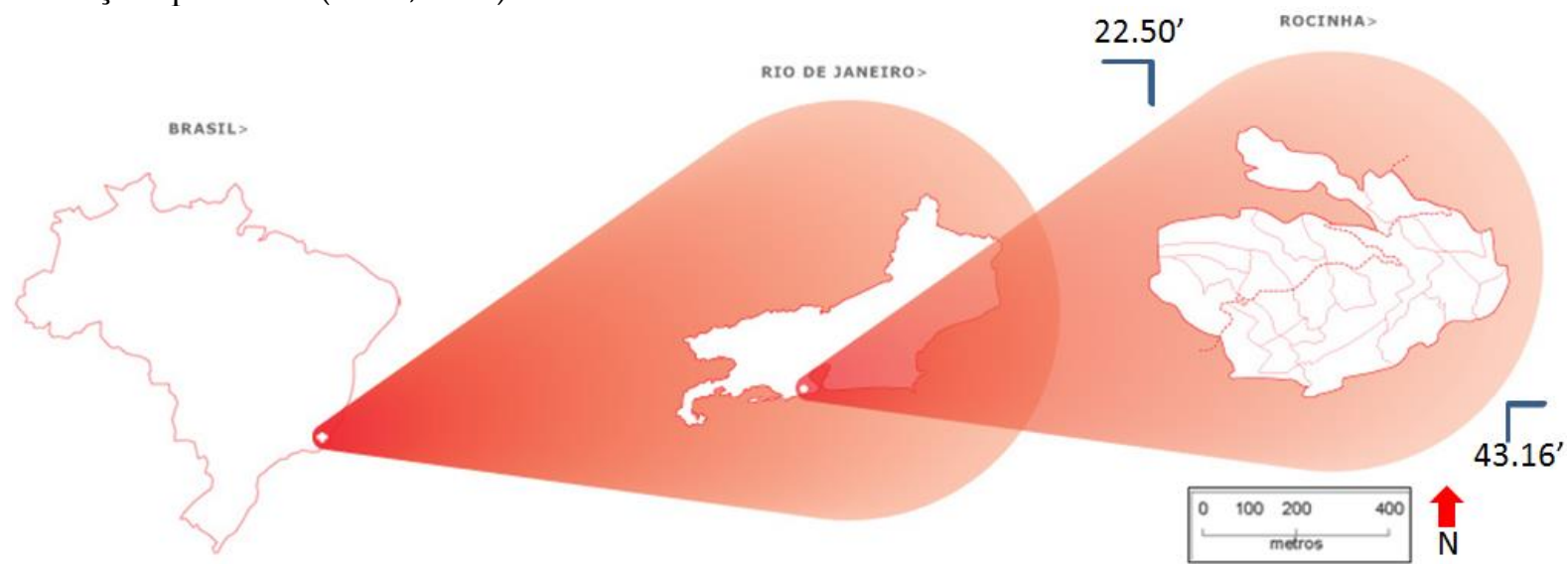

Figura 1 - Localização da Rocinha (RJ-Brasil).

Fonte: Mapa adaptado do Centro Internacional de Estudos e Pesquisas Sobre a Infância (2015).

Para atingir o objetivo proposto, inicialmente realizaram-se as seguintes etapas:

Etapa I - Revisão bibliográfica: possibilitou analisar diferentes opiniões de autores da área geomorfológica e áreas afins, dando maior ênfase à questão ambiental e ao conceito de ambiente (objeto de estudo). Tal revisão, segundo Siena (2007, p. 66), deve ser "elaborada ou desenvolvida a partir de material já publicado, em geral livros, artigos de periódicos e materiais disponibilizados na Internet".

Etapa II - Levantamento de dados de planialtimetria do terreno, georreferenciados, obtidos com o software Google Earth: Nesta etapa, foram coletados dados (longitude, latitude e altitude) de forma linear nos limites da área territorial da Rocinha, de forma interna, visando à representação real da topografia do terreno.

Assim, utilizou-se o Datum (modelo matemático teórico da representação da superfície da Terra ao nível do mar), WGS-84, representado no sistema projeção UTM (Universal Transverse Mercator). Na sequência, foram coletadas na área várias longitudes denominadas de Meridianos Centrais, localizando-se os pontos dentro do sistema terrestre.

Etapa III: Modelagem da topografia do terreno no software Surfer 12 a partir dos dados obtidos no Google Earth, e posterior extração de mapas e cortes planialtimétricos para avaliação das declividades e do potencial das áreas de risco de deslizamento: por meio desses dados, foram gerados (modelos) que, transformados em shape e sobrepostos no software Surfer 12, com análise de perfil de solo, permitiu a obtenção de dados referentes à declividade do local.

Etapa IV - Tempo de Concentração dos níveis pluviométricos: para diagnóstico de quanta água do escoamento pluviométrico da Rocinha acumula em minutos, foram utilizados os cálculos do tempo de 
concentração da equação de Vem Te Chow, considerando o acréscimo de um tempo de entrada de 5 minutos, até 5 casas decimais após a vírgula. Conforme a equação(1) de Mohtar (2015):

$$
t c=52,64\left(\frac{L}{\sqrt{S}}\right)^{0,64}
$$

Onde:

$L=$ Comprimento do talvegue $(\mathrm{km}) ; S=$ Declividade $(\mathrm{m} / \mathrm{km}) ; t c=$ Tempo de concentração $(\mathrm{min})$.

Etapa V - Diagnóstico da intensidade de chuvas na Rocinha: para obter-se a intensidade da chuva, foi utilizada a equação (2) de chuvas intensas, que se utiliza de dados pluviométricos em um determinado período de tempo. Conforme a equação (BACK, 2009, p. 172), tem-se:

$$
\begin{aligned}
& i=\frac{3445,7 T^{0,138}}{(t+26,3)^{1,012}} \\
& \text { Onde: } \\
& T=\text { Período de retorno (anos); } t \text { = Duração da chuva (minutos); } i=\text { Intensidade da precipitação (mm/h). }
\end{aligned}
$$

Através dessas etapas, torna-se possível a análise de riscos naturais da Rocinha (RJ), em relação à consequência geomorfológica do local.

\section{Resultados de Discussões}

A área ocupada pela Rocinha "possuía, originalmente, características rurais", segundo Leitão (2006, p. 5), já que "os primeiros registros apontam a existência no local, em meados da década de 1920, de uma grande fazenda, parcialmente ocupada, com a presença de uma densa floresta, contendo remanescente da Mata Atlântica". Consequentemente, durante os anos de 1927 até 1930, o local foi parcelado em lotes em torno de 270 metros quadrados, pela "necessidade do processo de urbanização da zona sul do Rio de Janeiro" (RJ).

Os habitantes dos aglomerados subnormais da Rocinha geralmente são pessoas de baixo poder aquisitivo (LEITÃO, 2006) que, geralmente, não fazem parte das estatísticas econômicas positivas do Brasil. Em 1990, ocorreu, no local, a verticalização das residências existentes, o que fez aumentar a situação de risco natural pelos possíveis solapamentos das encostas, pois esses aglomerados proporcionam o aumento da carga (peso) sobre a superfície íngreme do solo, tornando-se mais preocupante em épocas de intensas precipitações (ROBAINA, PEDROSA, BAZZAN, RECKZIEGEL, 2011).

Atualmente, a Rocinha ocupa uma área territorial de $453.440 \mathrm{~m}^{2}$, entre as encostas dos morros Dois Irmãos e Laboriaux (LEITÃO, 2004; LEITÃO, 2006). Assim, a Rocinha está estruturada sobre duas vertentes (interflúvio) com a altitude de 200 metros, que se ligam formando uma região de vale (talvegues) estreito de 0,22km (FIGURA 2), o que possibilita grande concentração de umidade. Isso torna o microclima urbano com maior temperatura do que a normalidade em épocas de radiação solar intensa e permite pouco escoamento da água em períodos chuvosos. 


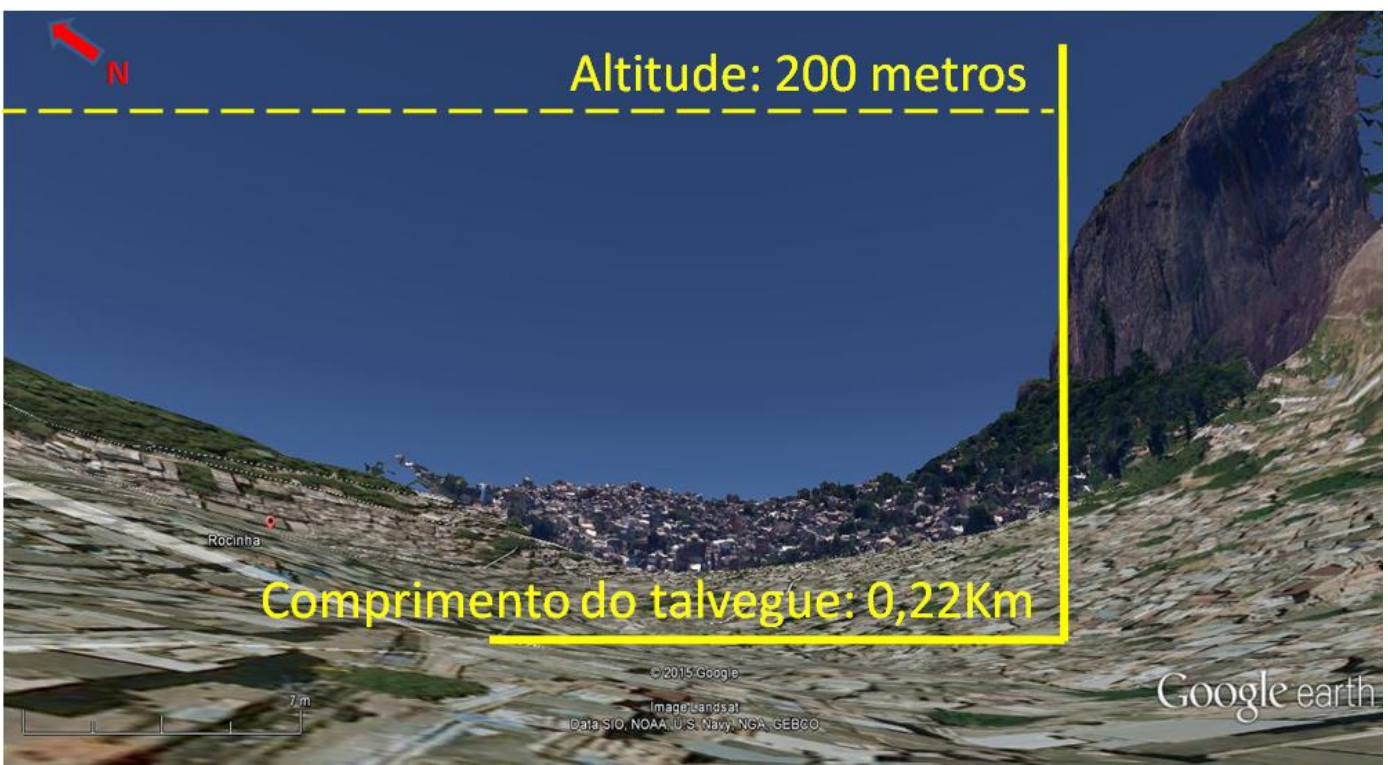

Figura 2 - Perfil representativo da Rocinha (RJ-Brasil).

Fonte: Imagem adaptada do Google earth (2015).

Em relação ao tempo de concentração $(t c)$ relacionado em minutos, o comprimento do talvegue principal $(L)$ (aproximadamente igual ao comprimento do curso d'água principal em $\mathrm{Km}$ ) é igual a 200 metros, enquanto a declividade $(S)$ de " $L$ " $(\mathrm{m} / \mathrm{km})$ é igual a 909,09091. Tais características permitem o diagnóstico do potencial de tempo (minutos) que a água de chuva que escoa das regiões de interflúvio leva para escoar ou infiltrar pelas regiões de talvegue da Rocinha.

$$
\begin{aligned}
& t c=52,64 \cdot\left(\frac{200}{\sqrt{909,09091}}\right)^{0,64} \\
& t c=52,64 \cdot\left(\frac{200}{30,15113}\right)^{0,64} \\
& t c=52,64,3,35664 \\
& t c=176,7 \mathrm{~min}
\end{aligned}
$$

Através da aplicabilidade do modelo matemático de Vem Te Chow, pode-se perceber que a equação sobre o tempo de concentração de escoamento da água foi de 176,7 (min). A Figura 3 demonstra que a representação do perfil planialtimétrico revelou uma declividade de forma acentuada, variando em 200 metros de altitude pela proximidade das isolinhas (curvas de nível), o que caracteriza a grande inclinação das vertentes. 


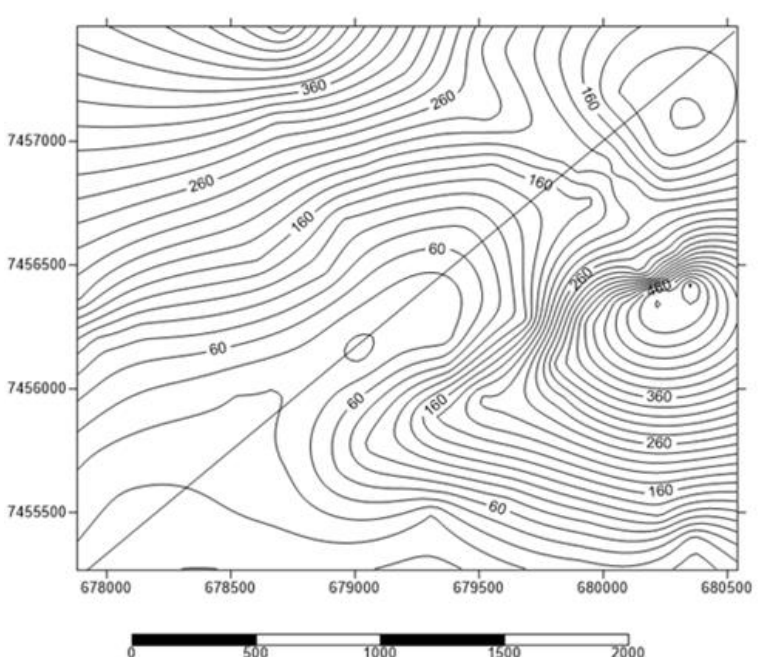

Figura 3 - Perfil planialtimétrico da Rocinha (RJ-Brasil).

Fonte: Imagens geradas no Software Surfer 12 (2015).

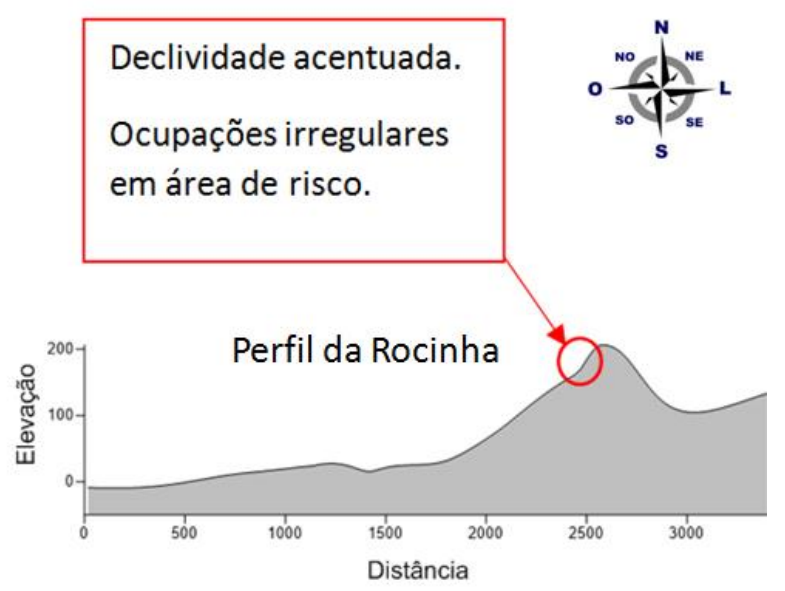

Quando aplicado o modelo matemático de chuvas intensas para estimar, em um determinado período de tempo, qual seria a Intensidade da precipitação (i), cujo Período de retorno $(T)$ é igual a 10 anos e a Duração da chuva ( $t$ ) é referente a 60 (minutos), soma-se um total de 52,00 (mm/h).

$$
\begin{aligned}
& i=\frac{3445,7.10^{0,138}}{(60+26,3)^{1,012}} \\
& i=\frac{3445,7.1,37404}{(86,3)^{1,012}} \\
& i=\frac{4734,52963}{91,04223} \\
& i=52,00 \mathrm{~mm} / \mathrm{h}
\end{aligned}
$$

Logo, a Intensidade da precipitação (i) (BACK, 2009, p. 172), em uma estimativa de 10 anos, revelou 52,00 mm/h da Rocinha. Quando se relaciona com o Tempo de concentração $(t c)$ de Vem Te Chow, percebe-se que o escoamento de água foi de 176,7(min). Ao integrar essa relação de modelagem, percebe-se que, daqui a 10 anos, essa quantidade de água precisaria de $(203,88 \mathrm{~min}) 3 \mathrm{~h} 39 \mathrm{~min}$ para escoar para fora da região de talvegue.

Conforme o Relatório Anual de Chuva para a cidade do Rio de Janeiro no ano de 2014, em relação à estimativa média mensal dos pluviômetros $(\mathrm{mm})$ do Sistema Alerta Rio entre 1997 e 2013, foi identificada uma média mensal de 143,3mm, que concentrou uma média anual de 1720,0mm no Aglomerado subnormal da Rocinha (D'ORSI, PAES, MAGALHÃES, COELHO, JUNIOR, CARNEIRO, 2015). As pesquisas de Robaina, Pedrosa, Bazzan e Reckziegel (2011, p. 22) relatam que a incidências constantes de precipitações podem afetar de forma direta a estrutura dos taludes, provocando problemas de "ocupação precária, desorganizada espacialmente e desprovida de infraestrutura adequada".

Nesse sentido, na Figura 4 podem ser visualizadas a Rocinha e sua área de risco, selecionada em cor diferenciada. Essa área representada foi definida em razão da identificação de pontos com maior altitude. Justifica-se, assim, a importância de demarcar esses espaços de risco de forma técnica, considerando alguns fatores (altitude, precipitação e volume pluviométrico acumulado). Segundo Robaina, Pedrosa, Bazzan e Reckziegel (2011, p. 22), a "falta da percepção do risco por parte das pessoas que vivem no local, baseada no fato do tempo que habitam a escarpa e o número de ocorrência de acidentes". 

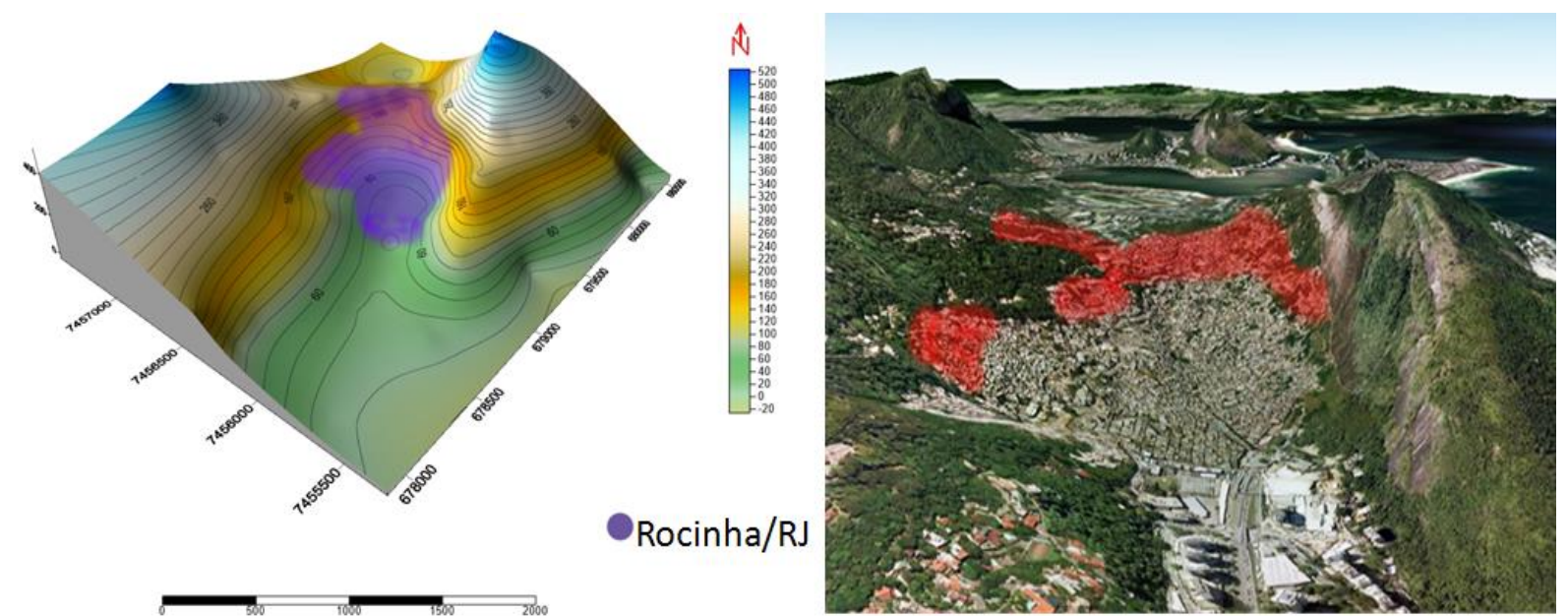

Figura 4 - Áreas de riscos geomorfológicos da Rocinha (RJ-Brasil).

Fonte: Imagens geradas no Software Surfer 12 e adaptado do Google earth (2015).

\section{Conclusões}

Os aglomerados subnormais caracterizam-se por um rápido crescimento demográfico. Esse fenômeno é consequência da atual condição econômica do Brasil, tornando essas ocupações uma saída para algumas pessoas que não têm condições de habitar outro lugar.

Entretanto, esse sistema habitacional esconde perigos à vida humana causados por questões físicas do terreno. Assim, em razão da declividade do terreno, não se deveria acumular residências em um perfil de solo que não se estabiliza, já que se trata de uma estrutura arenosa apoiada sobre rochas magmáticas densas.

A avaliação da topografia do terreno da Rocinha, pelo uso dos dados georreferenciados possibilitou a identificação das encostas de maior risco de deslizamento. A análise desses dados possibilita a realização de alertas, o que exige agilidade na tomada de decisão quanto a possíveis retiradas da população de áreas críticas, uma vez que estão sujeitas a riscos naturais geomorfológicos.

Outro fator agravante identificado é que a Rocinha (RJ) recebe precipitações em abundância, o que vem a agravar os riscos de deslizamentos. Uma solução que se aponta para tal problema de forma imediata consiste na retirada dessas pessoas para outros locais, permitindo-lhes condições de moradia digna.

Posteriormente, após a retirada das famílias habitantes da região, indica-se para a Rocinha (RJ) um plano de recuperação e de gestão ambiental que controle o monitoramento do local. Nesse caso, sugere-se que o local se transforme em uma reserva de preservação ambiental de proteção federal.

\section{Agradecimentos}

Agradecimentos ao Núcleo de Estudos e Pesquisas em Mobilidade Urbana (NEPMOUR) da Faculdade Meridional (IMED), pelo apoio financeiro para elaboração da pesquisa.

\section{Referências}

ANDRADE, L. S. Espaço Público e Favelas: Uma análise da dimensão pública dos espaços coletivos não edificados na Rocinha. Tese de Doutorado. Programa de Pós-Graduação em Geografia - UFRJ, Rio de Janeiro, 2002.

BACK, Á. J. Relações entre precipitações intensas de diferentes durações ocorridas no município de Urussanga, SC. Revista Brasileira de Engenharia Agrícola e Ambiental, v. 13, n. 2, p. 170-175. 2009. 
CARMO, A. M. do.; SOUTO, M. V. S.; DUARTE, C. R.; MESQUITA, Á. F. Análise de Risco Ambiental à Erosão Gerada a partir de produtos de sensores remotos: MDE Topodata e Landsat 8. In: SIMPÓSIO BRASILEIRO DE SENSORIAMENTO REMOTO - SBSR, 17., 2015, João Pessoa-pb. Anais XVII Simpósio Brasileiro de Sensoriamento Remoto. João Pessoa-PB: SBSR, 2015. p. 5927 - 5934.

D'ORSI, R. N.; PAES, N. M.; MAGALHÃES, M. A.; COELHO, R. da S.; JUNIOR, L. R. da S.; CARNEIRO, T. S. Relatório Anual de Chuva para a cidade do Rio de Janeiro no ano de 2014. In: Relatório GEO-RIO/DEP/GPE - N. 001/2015. 2015. Disponível em: <http://alertario.rio.rj.gov.br/upload/rel2014.pdf>. Acesso em: 08 out. 2015.

CENTRO INTERNACIONAL DE ESTUDOS E PESQUISAS SOBRE A INFÂNCIA (Brasil) (Comp.). Identificação e Localização. 2015. Disponível em: $<$ http://www.ciespi.org.br/primeira_infancia/cartografia/cartografias/rocinha/historico-rocinha/rocinhaidentificacao-e-localizacao>. Acesso em: 08 out. 2015.

GOOGLE EARTH. Imagens da Rocinha (RJ - Brasil. In: Programa Google Earth, 2015.

GOLDEN SOFTWARE. Surfer version 12: surface mapping system. Colorado, USA: Golden Software, 2015. CD Rom.

IBGE. INSTITUTO BRASILEIRO DE GEOGRAFIA E ESTATÍSTICA (Brasil). Presidência da Republica (Comp.). Censo Demográfico: Aglomerados subnormais. $2010 . \quad$ Disponível em: $<$ http://biblioteca.ibge.gov.br/visualizacao/periodicos/92/cd_2010_aglomerados_subnormais.pdf>. Acesso em: 08 out. 2015.

IBGE. INSTITUTO BRASILEIRO DE GEOGRAFIA E ESTATÍSTICA (Brasil). Presidência da Republica (Comp.). Glossario. 2010. Disponível em: < http://censo2010.ibge.gov.br/materiais/guia-do-censo/glossario>. Acesso em: 08 out. 2015.

LEITÃO, G. Dos barracos de madeira aos prédios de quitinetes: Uma análise do processo de produção da moradia na favela da Rocinha, ao longo de cinqüenta anos. Tese de Doutorado. Programa de Pós-Graduação em Geografia - UFRJ, Rio de Janeiro, 2004.

LEITÃO, G. Transformações na estrutura sócio-espacial das favelas cariocas, ao longo dos últimos cinqüenta anos: a Rocinha como exemplo. SEMINÁRIO DE HISTÓRIA DA CIDADE E DO URBANISMO SÃO PAULO, 4., 2006, São Paulo-SP. Anais... São Paulo: SHCU, 2006. p. 1 - 20.

MARANDOLA JR., E.; MARQUES, C.; PAULA, L. T. de.; CASSANELI, L. B. Crescimento urbano e áreas de risco no litoral norte de São Paulo. Revista Brasileira de Estudos da População, v. 30, n. 1, p. 35-56. 2013.

MOHTAR, R. H. Ven Te Chow Memorial Lecture: Localizing water and food security. Water International, v. 40, n. 4 , p. 559 - 567. 2015.

PREFEITURA DA CIDADE DO RIO DE JANEIRO/ Secretaria Municipal de Urbanismo/ Instituto Pereira Passos. A nova classificação de favelas para o planejamento das políticas públicas. Conselho Estratégico de Informações da Cidade. Públicado em Ata da Reunião de 12 de setembro de 2012. Rio de Janeiro, 2012. 19p.

ROBAINA, L. E. de S.; PEDROSA, A. de S.; BAZZAN, T.; RECKZIEGEL, E. W. Análise geográfica de áreas de risco de movimento de massa na região metropolitana de Porto, Portugal. Revista Brasileira de Geomorfologia, Porto Alegre, v. 12, n. 2, p.15-23, mar. 2011.

SIENA, O. Metodologia da Pesquisa Científica: Elementos para Elaboração e Apresentação de Trabalhos Acadêmicos. Gepes. Ppgmad. Departamento de Administração, Porto Velho: Unir, 2007. 201 p.

VIANA, D. R.; AQUINO, F. E.; MUNOZ, V. A. Avaliação de desastres no Rio Grande do Sul associados a complexos convectivos de mesoescala. Sociedade \& Natureza. v. 21, n.2, p. 91-105. 2009. 